\title{
The scope and extent of literature that maps threats to species: a systematic map protocol
}

\author{
Francesca Anne Ridley* (D, Philip JK McGowan and Louise Mair
}

\begin{abstract}
Background: The rate of anthropogenic biodiversity loss far exceeds the background rate of species extinctions. Global targets for biodiversity acknowledge this, nevertheless progress towards targets has been poor. There is now a reasonable understanding of what human pressures threaten the survival of species. However, information on where these threats are impacting species is needed to coordinate conservation actions and threat abatement efforts. Herein, threats are defined as human-driven pressures specifically where they co-occur with, and threaten the survival of, native wild species. There is a large number of studies that map either distributions of threatened species or human-driven pressures alone. This makes it difficult to identify research that has investigated the spatial distribution of the threats themselves. Additionally, the high variability in approaches taken in these studies promotes a high risk of duplication and diversity among the findings. This variation, and the lack of studies directly mapping threats, limits the utility of threat mapping studies for conservation planning and informing policy. Therefore, a systematic consolidation of the literature is necessary to identify where knowledge is lacking, and where sufficient evidence exists for synthesis of the collective findings.

Methods: This protocol details the process for a systematic mapping exercise aiming to identify studies that map threats to species across the world. For a study to be included it should present spatially explicit data on both the occurrence of species and the human-driven pressures threatening them. A range of peer-reviewed and grey literature repositories will be searched in English for literature published 2000-2020, followed by one iteration of backward snowballing. A three-stage screening process will be implemented before data are extracted on geographic coverage, taxonomic extent, and threats investigated. Data on the threats studied will be categorised using the threat classification scheme used by the IUCN Red List to allow comparisons among studies and to identify unrepresented threats. The extracted data will be analysed and visualised to describe the extent of existing knowledge. The resulting database of studies, findings from descriptive analyses, and accompanying narrative synthesis, will be made publicly available.
\end{abstract}

Keywords: Threat mapping, Biodiversity conservation, Evidence synthesis, Species extinctions, Human footprint

\section{Background}

Biodiversity is declining at a rate 100 to 1000 times the background rate [1], fast approaching that of a mass extinction event [2]. Furthermore, the body of evidence to support the reliance of people on biodiversity is extensive [3], with ecosystem services having an estimated

*Correspondence: f.ridley1@ncl.ac.uk

School of Natural and Environmental Sciences, Ridley Building 2,

Newcastle University, Newcastle upon Tyne NE1 7RU, UK worth of US\$125 trillion per year [4,5]. Multiple global targets acknowledge the need to combat threats to species in order to achieve biodiversity outcomes [6]. However, despite considerable progress made towards targets on protected land and sea areas, progress towards threatspecific targets has been poor, and species extinction rates continue to increase [3].

The threats to species around the world are numerous and diverse. Eighty percent of threatened or nearthreated species are impacted by more than one threat, 
the most frequent in IUCN Red List assessments was found to be overexploitation and agriculture $[7,8]$. Urban development, invasive species or disease, pollution, natural system modification, and climate change are also prevalent contributors to species' extinction risk [7, 8]. Many of these activities result in land-use change, which has been found to have the highest relative impact on terrestrial and freshwater ecosystems [3]. Threats to species are not distributed homogenously across the world. Schulze et al. [9] found that fire system modification was the most frequently reported threat to African protected areas, in contrast to Eurasia and North America where the most frequently reported threats were recreational activities and invasive species respectively. In addition, experts estimated that coral reef ecosystems were most heavily impacted by climate change, over-fishing, and pollution [10]. Meanwhile, freshwater ecosystems are subject to a suite of disruptions including water system modification, invasive species, and direct habitat loss due to the drainage of wetlands for development [11]. Therefore, information not only about what processes threaten species but how they are distributed spatially, is needed to identify and prioritise conservation actions.

\section{The purpose of threat maps}

Visualisations of the spatial distribution of threats to species (threat maps) have multiple, interrelated purposes that are closely linked to the spatial scale at which they are implemented. Threat maps are highly recommended in systematic conservation planning for deciding which actions to take where, and to prioritise limited resources $[12,13]$. Such plans tend to be localised and accompanied by techniques such as multi-criteria decision-making and cost-effective analysis $[14,15]$. In a similar way, at national, international and global scales threat maps support conservation policies and projects by engaging with policy officials and the public for the provision of awareness and funding $[13,16]$. For example, the Critical Ecosystem Partnership Fund has awarded over US\$231.9 million in grants specifically to conservation projects in Biodiversity Hotspots since 2000 [17, 18]. Eighty percent of threatened species rely on either conservation at multiple sites, or a combination of site and landscape scale action [19]. Therefore, all scales of threat mapping application, from local to global, are vital to preventing species extinctions.

A widespread conservation strategy with strong political traction is the demarcation and management of protected areas. This has variable effectiveness on conservation outcomes depending on whether the underlying threats are concurrently addressed [20-23]. Often overexploitation continues in protected areas despite political and legal restrictions [24-26]. However, the strict no-extraction policies recommended for optimum protected area management [27], are unfeasible where human communities rely on natural resources for their basic wellbeing [28]. Furthermore, many threatened species are widely distributed outside protected areas [2933]. Therefore, the knowledge of the spatial distribution of threats to species, both inside and outside protected areas is required to aid effective reserve management and inform wider landscape-scale conservation.

\section{Redefining threat mapping}

The range of terms used in the literature makes it difficult to pinpoint research that explicitly links the spatial distribution of threats to where species occur. The term 'threat mapping' has been broadly used to describe any spatial representation of the distribution, intensity or consequences of threat. Therefore, it has been used to refer to anything from hotspots of threatened species richness, or extinction risk categories [16, 22, 34], to the distribution of human-driven pressures irrespective of species presence (e.g. The Human Footprint Index $[35,36])$. For example, of four global prioritisation frameworks that included a measure of threat ([16] biodiversity hotspots [17], last of the wild [35], Crisis ecoregions [37], and High biodiversity wilderness areas [38]), none showed where species and threatening human activities co-occurred. Meanwhile, Di Marco et al. [39] represents a valuable advancement in threat mapping research, yet also does not provide this information. Their predictions for the probability of species persistence were based on betadiversity and abiotic environment rather than threatening human activities. Contrastingly, Allan et al. [40] combined data on human-driven pressures from the Human Footprint Index with threat and species distribution data from the IUCN Red List, revealing areas of active threat and refuges from threat. Furthermore, some studies use alternative words such as 'stress', 'impacts', or 'footprints' to describe threats to species [40-42], making it easy to overlook relevant evidence. Therefore, a revision in terminology is useful here, in order to distinguish maps that show the spatial coincidence of species and their threats from other representations of threat.

Part of the variability in terminology use comes from the fact that, although previous definitions distinguish between stress and impacts, pressures and threats are generally considered synonymous. Salafsky et al. [43] considered threats (or pressures) to be the direct human activities or processes that result in stress to species, where stress is a change in a species' ecological environment beyond normal levels of variation that compromises its survival. High densities of threatened species do not occur in every location where humans exert pressure on the landscape $[40,44]$. Meanwhile, some species are 
tolerant to human pressure [45]. Therefore, here 'pressures' are considered to be the human activities and direct human-initiated processes that have the potential to become 'threats' where they come into contact with wild species. Consequently, for the sake of this study, threat mapping research is considered that which describes or analyses the spatial distribution of threats to species. In other words, the spatial coincidence of wild populations of species and the human-driven pressures threatening them.

As the main purpose of threat mapping is to understand where threats occur, the findings must be projected onto a geographic distribution and presented spatially. Consider a study that investigates the effect of a threat on a species at a particular study site by gathering data on pressure intensity $(\mathrm{x})$ and species abundance $(\mathrm{y})$. If the study tested for a relationship between $\mathrm{x}$ and $\mathrm{y}$ without presenting a georeferenced spatial visualisation of how they varied across the study site, it would not be considered a threat mapping study. This is because the study itself did not present data on where geographically the species and human-driven pressure co-occurred.

\section{Identifying the gaps}

Consolidation of the threat mapping literature is a vital next step towards understanding the distribution of threats to species globally. A high diversity of approaches are present in the existing literature; varying in spatial extent, number and types of threats included, data types used, and specific questions asked [40, 41, 46-52]. A diverse and fragmented literature base presents a high risk of duplication of research effort and diversity among the findings, as shown in related fields. For example, Orme et al. [53] found low agreement among the findings of different biodiversity prioritisation frameworks. Meanwhile, Mace et al. [54] called for collaboration in light of the already high levels of duplication in conservation prioritisation efforts. Therefore, the likelihood of duplication and contradictory findings within the, yet uncharacterised, threat mapping literature is high. This would compromise a major purpose of threat maps, to prioritise limited resources for threat abatement action and awareness. Therefore, there is a need to locate gaps in knowledge, and identify where sufficiently similar evidence exists to facilitate synthesis of the collective findings.

It is likely that not all threats are equally represented in the literature. Threat mapping largely relies on satellite data and this reliance creates inherent biases in the types of threats considered in the existing literature [35, 36, 47]. For this reason, threats such as overexploitation are likely to be underrepresented in spatial analyses of threats [55], despite being the most frequently reported threat in IUCN Red List assessments [7]. In addition, Joppa et al. [8] found that biological resource use accounted for only $5 \%$ of datasets on species threats globally. Furthermore, despite often being studied spatially [56-58], invasive alien species were only included in three cumulative threat assessments found in preliminary literature searching $[40,59,60]$. To the authors' knowledge no systematic review of the literature analysing threats to species has been completed. Therefore, this work has significant potential to highlight gaps in knowledge of high benefit to threat abatement efforts.

This protocol describes the procedure that will be taken in the systematic map to follow, which will aim to collect and characterise the existing threat mapping literature. Systematic mapping is an exercise of rigorously and transparently gathering information from the existing literature, and describing it using data visualisation and analysis tools. In contrast to a systematic review, which collects and synthesises data on the findings, systematic maps tend to investigate the scope and extent of the literature base [61]. Previous applications in biodiversity conservation include: the impacts of conservation actions on human wellbeing [62], outcome reporting in systematic conservation planning [63], and the contribution of invasive species to biodiversity loss [64].

This systematic map is intended to fill a knowledge gap that has emerged during a wide range of discussions with diverse stakeholders during the development of the Post2020 Global Biodiversity Framework. As the negotiations move towards an emphasis on biodiversity outcomes for species as a high-level goal and action targets that will address the pressures/threats on species, it is expected that the findings from this map will inform these negotiations and implementation of the Framework when it is agreed.

\section{Objectives of the review}

This work aims to describe the threat mapping literature by analysing data on the methodological, geographic, and taxonomic extent of existing studies that map the threats to species. Gaps and clusters in knowledge will be identified, and a searchable database of threat mapping studies with corresponding meta-data will be created. Historic threat distributions are outside the scope of this study, therefore only studies published in the previous 20 years will be included. Similarly, work projecting future distributions of threats and studies that are purely modelling, theoretical or lab-based will be excluded. Another possible approach to studying where threats occur could be to study the spatial extent of all existing literature studying threats. However this is outside the scope of this investigation. 


\section{Primary question}

The primary overarching question of this analysis is: What is the scope and extent of literature that maps threats to species?

\section{Population}

The taxonomic scope of this study is any species of animal or plant globally, in any country or ecozone (terrestrial, marine, and freshwater). Species presence must be evidenced for studies to be included in the analysis. This evidence could be the observed range of individual species, or a pre-defined priority area for biodiversity conservation, such as a Biodiversity Hotspot (see Additional file 1). A pre-defined priority area is defined as one that has been identified to be of conservation priority in previous work by other authors or organisations, due to its presence, richness, or density of species. Habitat suitability or other modelled species distributions can be used provided there is evidence of species presence within the study site.

\section{Outcome}

The outcome to be examined is the spatial distribution of threats to species. By our definition, threats occur where species are exposed to human-driven pressures. Thus, the focus here is on the distribution of threatening human activities and direct human-initiated processes rather than the ecological mechanisms (stressors) that subsequently impact the state of species (for examples see Additional file 1). This is because one stressor can be caused by multiple interrelated human or natural processes. For example, a spatially explicit investigation into the concentration of manufactured pesticide and pharmaceutical contaminants in waterways that are protected for biodiversity conservation, would qualify for inclusion [65]. Whereas, an investigation measuring water quality indicators, without evidence of their anthropogenic source, would not be included [66]. Likewise, an investigation that observed species habitat conversion to specific human land-uses would qualify for inclusion. However, a study on habitat loss, with no further indication of the distribution of human activities responsible, would not be included. Studies measuring stress will only be included if the stress is explicitly a result of one or more threats and used as a proxy for those within the study.

To facilitate the consistent application of this definition throughout, the IUCN Red List threat classification scheme will be used as guide to categorise data on the threats studied in each article [67]. This hierarchical framework categorises threats to species into 12 high level categories which collectively encompass 103 fine scale categories of threat [67]. By doing so, threat mapping work using different methodologies and terminologies can be analysed together. Additionally, the use of the framework will reveal the proportional representation of different threats as well as any threats that are unrepresented within the literature base. Other classification schemes are more suited to capturing the complex interrelations between the activities, processes, and mechanisms driving species extinctions [68]. However, as the purpose of this investigation is to catalogue existing evidence, the IUCN framework was deemed the most appropriate for this.

\section{Study type}

This work will specifically identify studies that consider where the distributions of species and human-driven pressures coincide. Therefore, the population and the outcome component must be studied spatially within a global extent. The range of methodologies present in the literature complicates this. For example, if the distribution of pressures within a landscape is studied, and mean threat within protected areas is presented in a nonspatial sub-analysis, this would not merit inclusion (e.g. [22]). This is because it does not inform as to where the points of contact between protected areas and humandriven pressures are. Whereas, if studies take pre-existing knowledge about the threats affecting a particular species (such as from IUCN Red List assessments), and project it onto the geographic distribution of that species, these would be included.

\section{Secondary questions}

Further sub-group analyses will be structured around the following secondary questions:

1. What is the geographic distribution of the existing literature?

2. What is the taxonomic distribution of the existing literature?

3. Which threats are most frequently studied and how many different threats are considered in each study?

4. How has the extent of knowledge changed over time, and where do gaps and clusters in knowledge exist?

\section{Methods}

\section{Searching for articles}

Scoping

Fifteen relevant papers were chosen from preliminary reading to comprise the 'test-list' to develop the search and compare the comprehensiveness of searches against [69]. These were chosen based on their ability to capture the scope and diversity of terminology in the literature 
base (Additional file 2). Keywords were extracted along with unofficial keywords present in the title and abstract of these papers where designated keywords were unavailable. The keywords were grouped into terms and a thesaurus [70] used to identify appropriate synonyms (Additional file 2). The search string was developed and tested using SCOPUS, with the minimum combination of broad terms required to yield the full test-list of articles used as a starting point. Generality of the search was increased by adding synonyms, while specificity was increased by substituting broad terms for specific alternatives (Additional file 3). The exclusion rates of two candidate searches were compared by performing a titlelevel screen to determine the final search string (Additional file 2).

\section{Comprehensiveness of search}

The comprehensiveness of each individual search attempted was tested against its ability to return the testlist of fifteen benchmark articles (see Additional file 3). This was done to ensure that all searches considered met the baseline threshold for comprehensiveness. The first candidate search tested yielded 5679 articles in SCOPUS, of which $54 \%$ were excluded after a title-level screen. Whereas, the second candidate search yielded 10,463 articles in SCOPUS, of which $57 \%$ were excluded. The latter search was chosen as the final search string because 1859 more potentially relevant articles were found, approximately four for every ten additional articles that were screened (Additional file 2). This was deemed to support the choice of this search string as a balance of specificity and sensitivity. The final search yielded 28,990 results across the five published and grey literature databases proposed (Additional file 2). Therefore, a more sensitive search was not considered.

The final search string will be used to search publication databases, search engines and grey-literature repositories in English for articles published between 2000 and 2020. This time period was chosen as current, rather than historic or future, threat distributions are the focus of this investigation. It is recognised that local-scale studies from non-English speaking countries are likely to be overlooked however, restricted translation resources mean the inclusion of multiple languages is not feasible.

\section{Search terms}

The final search string is as follows:

(pressure OR threat OR risk OR stress OR footprint) AND (species OR ecosystem OR wildlife OR fauna OR flora OR \{spp.\} OR \{sp.\}) AND (hotspot* OR map* OR geographic" OR "gis" OR "spatial distribution" OR "spatial overlap" OR "spatial separation" OR "spatial dynamics" OR "spatial variation" OR "spatial framework" OR "spatially explicit" OR geospatial) AND (conservation OR biodiversity).

\section{Publication databases to search}

SCOPUS, ProQuest natural science collection, and Web of Science Core collection will be searched for published peer-reviewed studies by title, abstract, and keywords. The final search performed using these three databases collectively yielded 28,097 articles $(10,462,8859$, and 8776 respectively), of which 13,933 were found to be unique. As the number of unique results is greater than any single search, all three will be used to search for published literature. All searches will be performed using the subscription of Newcastle University. For specifics of the subscription and how each database will be searched see Additional file 2.

\section{Search engine}

Google Scholar will be used to identify grey literature using the simplified search of: (pressure OR threat OR footprint) AND (species OR ecosystem OR wildlife). The title only will be used to search for literature on google scholar as this has been found to be more efficient than searching the full text [75]. The first 500 results will be gathered in order of relevance.

\section{Grey literature searches}

To find grey literature ProQuest Natural collection will be specifically searched for non-peer reviewed Dissertations \& Theses, Government and official publications, reports, and working papers using the same search string as the published literature search.

\section{Organisational websites}

The following organisational websites will be searched using the word 'threats. The word 'threats' resulted in the same or more results than the word 'threat' across almost all websites. The only exception was Blue Ventures [71] for which 'threat' returned more, and so this website only will be searched using the word 'threat' instead.

- WWF, World Wildlife Fund [72].

- UNEP-WCMC [73].

- CBD, Convention on biological Diversity [74].

- IUCN, International Union for the Conservation of Nature [75].

- IPBES, Intergovernmental Panel on Biodiversity and Ecosystem Services [76]

- RSPB [77]

- Fauna and Flora International [78]

- The Nature Conservancy [79]

- Conservation International [80]

- Birdlife International [81] 
- Blue Ventures [71]

- The Audubon Society [82]

- SCB, Society of Conservation Biology [83]

\section{Targeted searches}

Backward snowballing will be used to identify additional studies, whereby the literature cited in all articles included after full-text screening will be collected. Previous work has found this technique to be twice as effective in identifying relevant articles as database searching [84]. The same procedure of duplicate removal and screening will then be applied to these articles.

\section{Article screening and study eligibility criteria Screening process}

A three-stage screening process (Title, Abstract, and Full-text) will be undertaken using EPPI-reviewer 4 [85]. At each stage, articles will be compared against the eligibility criteria below. At the title stage if there is insufficient information presented to conclusively exclude a study, all studies pertaining to an effect of human-driven threats on species, or prioritising conservation efforts, will be screened again at abstract level. If at the abstract stage there is insufficient information to categorically exclude a study based on the eligibility criteria, it will be retained to the full-text stage. A record will be kept of all studies excluded at the full-text screening stage, reporting the reasons for their exclusion.

\section{Consistency checking}

The screening process will be predominantly carried out by one core reviewer. At each screening stage (title, abstract, and full-text) a random $20 \%$ of articles, to a minimum of 50, will be selected for second review and Kappa analysis. Cohen's Kappa will be used to calculate the proportional agreement between the two reviewers with two outcome categories 'Include' or 'Exclude', and accounting for the agreement expected by chance [86]. Any disagreements will be discussed between reviewers and a decision whether to include or exclude the articles made. If the resulting kappa statistic is less than 0.6, the robustness of the eligibility criteria will be re-considered [87]. None of the reviewers are expected to author any articles to be considered within the review. However should the situation arise, this will be declared and detailed reasoning for all decisions regarding the articles in question will be reported.

\section{Eligibility criteria}

1. Eligible population: The taxonomic scope of this study is any species of animal or plant globally, in any country or ecozone (terrestrial, marine, and freshwater). Species presence must be evidenced for studies to be included in the analysis. This evidence could be the known range of species, or a pre-defined priority area for biodiversity, such as a protected area or Biodiversity Hotspot. In all instances where a predefined metric is used, the presence of species must be a qualifying criteria of that definition for the study to be included. Examples of protected areas and priority areas for biodiversity that do, and do not, meet this criteria are given in Additional file 1. Similarly, habitat suitability or other modelled species distributions can be used provided there is evidence of species presence within the modelled distribution.

a. Excluded if: No evidence is given for the distribution or presence of relevant species within the study area.

b. Excluded if: The study models habitat suitability on purely ecological factors without evidence of species presence at the study site e.g. for the purpose of reintroduction.

2. Eligible outcome: the spatial distribution of threats to species. Threats occur where species populations are exposed to human-driven pressure. Whereby, human-driven pressures are the activities and human-initiated processes that have the potential to negatively impact the ecological fitness of species. Where studies include multiple 'threats', at least one must fit the definition of a threat used here for the study to be included. If the threats included fit the definition of threats used here but cannot be assigned to one of the IUCN threat classification scheme categories, then "Other" will be assigned. The distribution of multiple threats can be presented individually or as a composite index. Where invasive alien species are considered, the distribution of the invasive alien will be considered the threat distribution.

a. Excluded if: No data on where species and humandriven pressures co-occur is presented e.g. measuring threatened species richness.

b. Excluded if: ecological stress is measured without being explicitly considered a valid proxy for a specific threat.

c. Excluded if: the threat considered is listed in the IUCN threat classification scheme but is not necessarily a result of human action (e.g. geological events). Studies on threats such as fire, extreme weather, and disease, should only be included if they are specifically human-induced in the context of the study. 
3. Eligible study types: only primary research published 2000-2020 in English will be included, which may use either primary data, or pre-existing datasets. Exceptions can be made in the case of systematic reviews and meta-analyses where data from primary work has been collated and re-analysed. Only studies assessing current threat distributions will be included, as opposed to projected future distribution or historic distributions.

a. Excluded if: The article is a narrative review in which no new synthesis of data is being presented.

b. Excluded if: The displayed distribution is projected forward in time or describing historic threat distributions.

c. Excluded if: The threat distribution is not within a global spatial extent whether theoretical or due to insufficient reporting.

\section{Study validity assessment}

Validity of individual studies will not be assessed beyond study eligibility based on the above criteria. Information on the study designs and data types used will be coded, allowing for future assessment of the validity of those methods.

\section{Data coding strategy}

Coding of the articles included at the full-text stage will be completed by filling in a pre-designed data collection sheet with discrete fields for data entry, for which McIntosh et al. [63] has been used as a template. The data sheet was tested using the fifteen-article test-list to assess usability and clarity (see Additional file 4). $20 \%$ of articles will be randomly chosen to be coded by a second reviewer. Any discrepancies will be discussed, and decisions about the correct coding to apply will be made collaboratively between reviewers.

Data will be extracted from the articles on bibliography, study design, threat measurement, and population studied. Data collected must be presented within the article or the additional material to be coded. Authors will not be contacted regarding missing information. The IUCN Red List threat classification scheme will form the typology for coding data on the number of, and which specific threats were studied in each article. Of these the twelfth high level category is "Other" and therefore, all humandriven threats will be accounted for. Information relating to population will be gathered with regards to the measure for species presence used, in addition to the taxonomic extent and resolution.

\section{Study mapping and presentation}

The findings will be published in this journal along with a searchable database of articles and the corresponding coded meta-data. Detailed information on the flow of articles through the process will be included, including the number of papers acquired from each source and those excluded at each stage. A geographic map will be produced detailing the coverage and density of study areas across the world, also displaying the distribution of studies among different spatial scales.

The taxonomic distribution of existing research will be visualised by plotting taxonomic groups against taxonomic resolution, whereby the response value will be the number of studies satisfying the two conditions. This will highlight if, for example, most studies on threats to plants take a kingdom wide approach, whilst most studies on mammals are family or genus specific. The mean number of threats considered per study will be presented with variance given, in addition to frequencies of occurrence of each threat category. Linkages between spatial distribution, taxonomy, and threats studied will be evaluated using co-occurrence matrices. The report will be complemented by a narrative synthesis discussing the range of approaches implemented, and avenues for future research.

\section{Supplementary information}

Supplementary information accompanies this paper at https://doi. org/10.1186/s13750-020-00206-8.

Additional file 1. Eligible population and outcome: Examples of predefined areas that are considered eligible proxies for population, and examples of eligible threats.

Additional file 2. Search strategy: Details of search strategy development including the test-list of benchmark articles, identification of keywords, development and testing of search strings, and specifics of the final search for each database used.

Additional file 3. Search testing specifics: Details of every search attempted in the process of search string development in SCOPUS.

Additional file 4. Data Coding Tool: The spreadsheet used for coding of meta-data, including descriptions of the topography and definitions to be used by reviewers.

Additional File 5. completed ROSES Form.

\section{Acknowledgements}

Many thanks to Professor Steven Rushton for his input in regard to refining the systematic map methodology and manuscript. Thanks also to Emily Hickinbotham, who will act as the second reviewer in the systematic map process. LM acknowledges the support of Newcastle University and the IUCN Species Survival Commission Conservation Planning Specialist Group.

\section{Authors' contributions}

FAR conceived and designed the systematic map and drafted the manuscript. LM made adjustments to the methodology, including to the inclusion/exclusion criteria that resulted in the final protocol design. LM and PM refined the scope, terminology, and structure of the manuscript. All authors read and approved the final manuscript. 


\section{Funding}

FAR is supported by a OnePlanet PhD studentship funded by UKRI via the Natural and Environmental Research Council. Grant number [NE/S007512/1].

\section{Availability of data and materials}

All data analysed in the formulation of this protocol is available via the databases cited and the additional material.

\section{Ethics approval and consent to participate}

Not applicable.

\section{Consent for publication}

Not applicable.

\section{Competing interests}

The authors declare that they have no competing interests.

Received: 27 April 2020 Accepted: 17 September 2020

Published online: 23 September 2020

\section{References}

1. Pimm SL, Jenkins CN, Abell R, Brooks TM, Gittleman JL, Joppa LN, et al. The biodiversity of species and their rates of extinction, distribution, and protection. Science. 2014;344:1246752.

2. Barnosky AD, Matzke N, Tomiya S, Wogan GO, Swartz B, Quental TB, et al. Has the earth's sixth mass extinction already arrived? Nature. 2011:471:51-7.

3. IPBES. Summary for policymakers of the global assessment report on biodiversity and ecosystem services of the intergovernmental science-policy platform on biodiversity and ecosystem services. Bonn: IPBES Secretariat; 2019.

4. Costanza R, De Groot R, Sutton P, Van der Ploeg S, Anderson SJ, Kubiszewski I, et al. Changes in the global value of ecosystem services. Global Environ Change. 2014;26:152-8.

5. WWF. Living planet report-2018: Aiming higher. Gland, Switzerland; 2018

6. CBD. Cop 10 decision x/2:Stategic plan for biodiversity 2011-2020. 2010.

7. Maxwell SL, Fuller RA, Brooks TM, Watson JE. Biodiversity: the ravages of guns, nets and bulldozers. Nature News. 2016;536:143.

8. Joppa L, O'Connor B, Visconti P, Smith C, Geldmann J, Hoffmann M, et al. Filling in biodiversity threat gaps. Science. 2016;352:416-8.

9. Schulze K, Knights K, Coad L, Geldmann J, Leverington F, Eassom A, et al. An assessment of threats to terrestrial protected areas. Conserv Lett. 2018;11:e12435.

10. Halpern BS, Selkoe KA, Micheli F, Kappel CV. Evaluating and ranking the vulnerability of global marine ecosystems to anthropogenic threats. Conserv Biol. 2007;21:1301-15.

11. Cazzolla Gatti R. Freshwater biodiversity: a review of local and global threats. Int J Environ Stud. 2016;73:887-904.

12. Margules CR, Pressey RL. Systematic conservation planning. Nature. 2000;405:243-53.

13. Tulloch VJ, Tulloch AI, Visconti P, Halpern BS, Watson JE, Evans MC, et al. Why do we map threats? Linking threat mapping with actions to make better conservation decisions. Front Ecol Environ. 2015;13:91-9.

14. Auerbach NA, Tulloch Al, Possingham HP. Informed actions: where to cost effectively manage multiple threats to species to maximize return on investment. Ecol Appl. 2014;24:1357-73.

15. Carwardine J, O'Connor T, Legge S, Mackey B, Possingham HP, Martin TG. Prioritizing threat management for biodiversity conservation. Conserv Lett. 2012;5:196-204.

16. Brooks TM, Mittermeier RA, da Fonseca GA, Gerlach J, Hoffmann M, Lamoreux JF, et al. Global biodiversity conservation priorities. Science. 2006:313:58-61.

17. Myers N, Mittermeier RA, Mittermeier CG, Da Fonseca GA, Kent J. Biodiversity hotspots for conservation priorities. Nature. 2000:403:853-8.

18. CEPF. Annual report 2018. Arlington; 2018.

19. Boyd C, Brooks TM, Butchart SH, Edgar GJ, Da Fonseca GA, Hawkins F, et al. Spatial scale and the conservation of threatened species. Conserv Lett. 2008;1:37-43.
20. Mora C, Sale PF. Ongoing global biodiversity loss and the need to move beyond protected areas: a review of the technical and practical shortcomings of protected areas on land and sea. Mar Ecol Prog Ser. 2011;434:251-66

21. Pfeifer M, Burgess ND, Swetnam RD, Platts PJ, Willcock S, Marchant R. Protected areas: mixed success in conserving east africa's evergreen forests. PLOS ONE. 2012;7:e39337.

22. Geldmann J, Joppa LN, Burgess ND. Mapping change in human pressure globally on land and within protected areas. Conserv Biol. 2014;28:1604-16.

23. Jones KR, Venter O, Fuller RA, Allan JR, Maxwell SL, Negret PJ, et al. Onethird of global protected land is under intense human pressure. Science. 2018;360:788-91.

24. Dureuil M, Boerder K, Burnett KA, Froese R, Worm B. Elevated trawling inside protected areas undermines conservation outcomes in a global fishing hot spot. Science. 2018;362:1403-7.

25. Golden CD. Bushmeat hunting and use in the makira forest, northeastern madagascar: a conservation and livelihoods issue. Oryx. 2009;43:386-92.

26. Golden HE, Knightes CD. Simulated watershed mercury and nitrate flux responses to multiple land cover conversion scenarios. Environ Toxicol Chem. 2011;30:773-86

27. Edgar GJ, Stuart-Smith RD, Willis TJ, Kininmonth S, Baker SC, Banks S, et al. Global conservation outcomes depend on marine protected areas with five key features. Nature. 2014;506:216-20.

28. Oldekop JA, Holmes G, Harris WE, Evans KL. A global assessment of the social and conservation outcomes of protected areas. Conserv Biol. 2016;30:133-41.

29. Rodrigues AS, Andelman SJ, Bakarr MI, Boitani L, Brooks TM, Cowling RM, et al. Effectiveness of the global protected area network in representing species diversity. Nature. 2004;428:640-3.

30. Dudley N, Jonas H, Nelson F, Parrish J, Pyhälä A, Stolton S, et al. The essential role of other effective area-based conservation measures in achieving big bold conservation targets. Global Ecol Conserv. 2018;15:e00424.

31. Dinerstein E, Olson D, Joshi A, Vynne C, Burgess ND, Wikramanayake E, et al. An ecoregion-based approach to protecting half the terrestrial realm. Bioscience. 2017;67:534-45.

32. Noss RF, Dobson AP, Baldwin R, Beier P, Davis CR, Dellasala DA, et al. Bolder thinking for conservation. Conserv Biol. 2012;26:1-4.

33. Magris RA, Pressey RL. Marine protected areas: just for show? Science. 2018;360:723-4.

34. Wilson KA, McBride MF, Bode M, Possingham HP. Prioritizing global conservation efforts. Nature. 2006;440:337-40.

35. Sanderson EW, Jaiteh M, Levy MA, Redford KH, Wannebo AV, Woolmer G. The human footprint and the last of the wild: the human footprint is a global map of human influence on the land surface, which suggests that human beings are stewards of nature, whether we like it or not. Bioscience. 2002;52:891-904.

36. Venter O, Sanderson EW, Magrach A, Allan JR, Beher J, Jones KR, et al, Global terrestrial human footprint maps for 1993 and 2009. Scientific Data. 2016:3:1-10.

37. Hoekstra JM, Boucher TM, Ricketts TH, Roberts C. Confronting a biome crisis: global disparities of habitat loss and protection. Ecol Lett. 2005:8:23-9.

38. Mittermeier RA, Mittermeier CG, Brooks TM, Pilgrim JD, Konstant WR, da Fonseca GA, et al. Wilderness and biodiversity conservation. Proc Natl Acad Sci. 2003;100:10309-13.

39. Di Marco M, Ferrier S, Harwood TD, Hoskins AJ, Watson JE. Wilderness areas halve the extinction risk of terrestrial biodiversity. Nature. 2019;573:582-5.

40. Allan JR, Watson JE, Di Marco M, O'Bryan CJ, Possingham HP, Atkinson SC, et al. Hotspots of human impact on threatened terrestrial vertebrates. PLoS Biol. 2019;17:e3000158.

41. Brown CJ, Saunders MI, Possingham HP, Richardson AJ. Interactions between global and local stressors of ecosystems determine management effectiveness in cumulative impact mapping. Divers Distrib. 2014:20:538-46.

42. Comte L, Olden JD. Climatic vulnerability of the world's freshwater and marine fishes. Nat Clim Change. 2017;7:718-22.

43. Salafsky N, Salzer D, Stattersfield AJ, Hilton-Taylor C, Neugarten R, Butchart $\mathrm{SH}$, et al. A standard lexicon for biodiversity conservation: unified classifications of threats and actions. Conserv Biol. 2008:22:897-911. 
44. Venter O, Sanderson EW, Magrach A, Allan JR, Beher J, Jones KR, et al. Sixteen years of change in the global terrestrial human footprint and implications for biodiversity conservation. Nat Commun. 2016;7:1-11.

45. Rosset V, Oertli B. Freshwater biodiversity under climate warming pressure: identifying the winners and losers in temperate standing waterbodies. Biol Cons. 2011;144:2311-9.

46. Chaudhary A, Brooks TM. National consumption and global trade impacts on biodiversity. World Dev. 2019;121:178-87.

47. Evans MC, Possingham HP, Wilson KA. What to do in the face of multiple threats? Incorporating dependencies within a return on investment framework for conservation. Divers Distrib. 2011;17:437-50.

48. Fuller D, Meijaard E, Christy L, Jessup T. Spatial assessment of threats to biodiversity within east kalimantan, indonesia. Appl Geogr. 2010;30:416-25.

49. Gaisberger H, Kindt R, Loo J, Schmidt M, Bognounou F, Da SS, et al. Spatially explicit multi-threat assessment of food tree species in burkina faso: a fine-scale approach. PLoS ONE. 2017;12:e0184457.

50. Gallardo B, Zieritz A, Aldridge DC. The importance of the human footprint in shaping the global distribution of terrestrial, freshwater and marine invaders. PLoS ONE. 2015:10:e0125801.

51. Parravicini V, Rovere A, Vassallo P, Micheli F, Montefalcone M, Morri C, et al. Understanding relationships between conflicting human uses and coastal ecosystems status: a geospatial modeling approach. Ecol Ind. 2012;19:253-63.

52. Moran D, Kanemoto K. Identifying species threat hotspots from global supply chains. Nat Ecol Evolut. 2017;1:1-5.

53. Orme CDL, Davies RG, Burgess M, Eigenbrod F, Pickup N, Olson VA, et al. Global hotspots of species richness are not congruent with endemism or threat. Nature. 2005;436:1016-9.

54. Mace GM, Balmford A, Boitani L, Cowlishaw G, Dobson AP, Faith D, et al. It's time to work together and stop duplicating conservation efforts.... Nature. 2000:405:393.

55. McGowan PJ. Conservation: mapping the terrestrial human footprint Nature. 2016;537:172-3

56. Clarke Murray C, Gartner H, Gregr EJ, Chan K, Pakhomov E, Therriault TW. Spatial distribution of marine invasive species: environmental, demographic and vector drivers. Divers Distrib. 2014;20:824-36.

57. Welk E, Schubert K, Hoffmann MH. Present and potential distribution of invasive garlic mustard (alliaria petiolata) in north america. Divers Distrib. 2002:8:219-33.

58. Zhu L, Sun OJ, Sang W, Li Z, Ma K. Predicting the spatial distribution of an invasive plant species (eupatorium adenophorum) in china. Landscape Ecol. 2007;22:1143-54

59. Halpern BS, Walbridge S, Selkoe KA, Kappel CV, Micheli F, D’Agrosa C, et al. A global map of human impact on marine ecosystems. Science. 2008;319:948-52.

60. Reddy CS, Khuroo AA, Krishna PH, Saranya K, Jha C, Dadhwal V. Threat evaluation for biodiversity conservation of forest ecosystems using geospatial techniques: a case study of odisha, india. Ecol Eng. 2014;69:287-303

61. James KL, Randall NP, Haddaway NR. A methodology for systematic mapping in environmental sciences. Environ Evid. 2016;5:7.

62. McKinnon MC, Cheng SH, Dupre S, Edmond J, Garside R, Glew L, et al. What are the effects of nature conservation on human well-being? A systematic map of empirical evidence from developing countries. Environ Evid. 2016;5:1-25

63. McIntosh EJ, Chapman S, Kearney SG, Williams B, Althor G, Thorn JP, et al. Absence of evidence for the conservation outcomes of systematic conservation planning around the globe: a systematic map. Environ Evid. 2018:7:22.
64. Roberts PD, Diaz-Soltero H, Hemming DJ, Parr MJ, Wakefield NH, Wright $\mathrm{HJ}$. What is the evidence that invasive species are a significant contributor to the decline or loss of threatened species? A systematic review map. Environ Evid. 2013;2:5.

65. Bradley PM, Romanok KM, Duncan JR, Battaglin WA, Clark JM, Hladik ML, et al. Exposure and potential effects of pesticides and pharmaceuticals in protected streams of the us national park service southeast region. Sci Total Environ. 2020;704:135431.

66. Zambrano L, Contreras V, Mazari-Hiriart M, Zarco-Arista AE. Spatial heterogeneity of water quality in a highly degraded tropical freshwater ecosystem. Environ Manage. 2009;43:249-63.

67. IUCN C. Unified classification of direct threats, version 1.0. World Conservation Union, The Conservation Measures Partnership Gland ; 2006.

68. Balmford A, Carey P, Kapos V, Manica A, Rodrigues AS, Scharlemann JP, et al. Capturing the many dimensions of threat: Comment on salafsky et al. Conserv Biol. 2009;23:482-7.

69. Booth A, Sutton A, Papaioannou D. Systematic approaches to a successful literature review: Sage; 2016.

70. Dictionary.com. Thesaurus.Com: Lexico Publishing Group (LLC); 2020 www.thesaurus.com

71. Ventures B. Blue ventures 2020 https://blueventures.org/.

72. WWF. Homepage World Wildlife Fund 2020 https://www.wwf.org.uk/.

73. UNEP-WCMC. Resources and data 2020 https://www.unep-wcmc.org/ resources-and-data.

74. CBD. Knowledge base: Convention on Biological Diversity 2020 https:// www.cbd.int/kb/.

75. IUCN. Homepage: Internation Union for Conservation of Nature 2020 https://www.iucn.org/.

76. IPBES. Homepage: Intergovernmental Panel on Biodiversity and Ecosystem Services 2020 https://ipbes.net/.

77. RSPB. Homepage: Royal Society for Protection of Birds 2020 https://www rspb.org.uk/.

78. Fauna-Flora. Homepage: Fauna and Flora International 2020 https://www. fauna-flora.org/.

79. Conservancy TN. Homepage: The Nature Conservancy; 2020 https:// www.nature.org/en-us/.

80. International C. Homepage: Conservation International; 2020 https:// www.conservation.org/.

81. International B. Homepage: BirdLife International; 2020 www.birdlife.org/.

82. Audubon. National audubon society: Audubon; 2020 https://www.audub on.org/.

83. SCB. Homepage: Society for Conservation Biology; 2020 https://conbi o.org/.

84. Badampudi D, Wohlin C, Petersen K, editors. Experiences from using snowballing and database searches in systematic literature studies. Proceedings of the 19th International Conference on Evaluation and Assessment in Software Engineering: 2015.

85. Thomas J, Brunton J, Graziosi S. Eppi-reviewer 4.0: Software for research synthesis. 2010

86. Cohen J. A coefficient of agreement for nominal scales. Educ Psychol Measur. 1960:20:37-46.

87. McHugh ML. Interrater reliability: the kappa statistic. Biochemia Medica. 2012:22:276-82.

\section{Publisher's Note}

Springer Nature remains neutral with regard to jurisdictional claims in published maps and institutional affiliations. 